\title{
Human-centred Mobile Pedestrian Navigation Systems ${ }^{1)}$
}

\author{
Georg Gartner, Haosheng Huang, Alexandra Millonig, Manuela Schmidt, \\ and Felix ORTAG, all Vienna*
}

with 5 Fig. in the text

\section{CONTENTS}

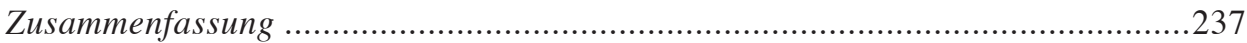

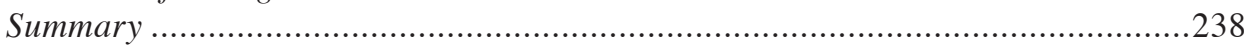

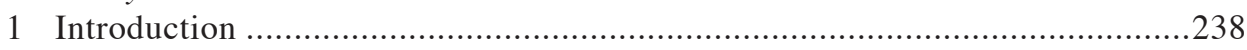

2 Human navigation and mobile navigation systems ...................................239

3 Selected contributions to the concept of human-centred mobile pedestrian navigation systems ...................................................241

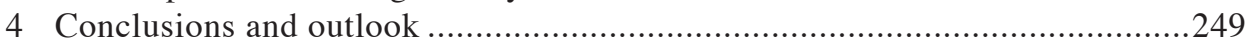

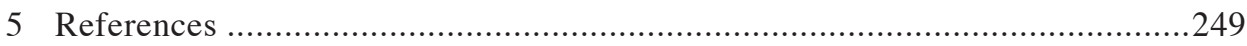

\section{Zusammenfassung}

Nutzerzentrierte mobile Fußgängernavigationssysteme

In fremden Umgebungen benötigen Menschen häufig Hilfe, um ein bestimmtes Ziel zu erreichen. Für diesen Zweck werden mobile Fußgängernavigationssysteme entwickelt. Derzeit wenden viele dieser Systeme Methoden der Autonavigation in adaptierter Form an. Forschungsergebnisse zeigen jedoch, dass sich der Informationsbedarf von

\footnotetext{
1) We would like to acknowledge the support of the Austrian FWF-funded project UCPNavi, FFG-funded project SemWay, and FFG-funded project ways2navigate. We also thank our partners, i.e. Engineering Geodesy Group at Vienna University of Technology, Arsenal Research (now Austrian Institute of Technology), Salzburg Research, FACTUM, TraffiCon and WalkSpace Mobilität, for their contributions to these projects.

* Univ.-Prof. Dr. Georg Gartner, Proj.-Ass. Haosheng Huang MSc., Dipl.-Ing. Alexandra Millonig, Univ.-Ass. Dipl.-Ing. (FH) Manuela SCHMIDT, and Univ.-Ass. Dipl.-Ing. Felix ORTAG, all Research Group Cartography, Vienna University of Technology, Gusshausstraße 30/1. Stock, A-1040, Vienna, Austria; e-mail: georg.gartner@tuwien.ac.at, haosheng.huang@tuwien.ac.at, alexandra.millonig@ait.ac.at, manuela. schmidt@tuwien.ac.at, felix.ortag@tuwien.ac.at; http://cartography.tuwien.ac.at/
} 
Fußgängern deutlich von jenem der Autofahrer unterscheidet. Daraus kann geschlossen werden, dass Entwicklungsansätze von Navigationssystemen für Autofahrer nicht geeignet sind, um auf Fußgängernavigation übertragen zu werden.

In Anbetracht der Herausforderungen wird ein Konzept vorgestellt, welches den Menschen in den Mittelpunkt rückt. Dabei werden Bedürfnisse und Einschränkungen von Fußgängern berücksichtigt, mit dem Ziel die Nützlichkeit von Fußgängernavigationssystemen zu optimieren. Dieser Artikel fasst die Ergebnisse aktueller Forschungsanstrengungen zu diesen Themen zusammen, wobei der Fokus auf Routenplanung und Routenkommunikation liegt.

\section{Summary}

People in unfamiliar environments often need assistance to reach a destination. Mobile pedestrian navigation systems are designed for this purpose. Currently, many pedestrian navigation systems still adopt the methods employed in car navigation systems. Research has shown that information needs of pedestrians are quite different from those of car drivers. Therefore, approaches related to car navigation systems are not appropriate for designing systems for pedestrians.

In recognizing the challenges, we propose the concept of human-centred pedestrian navigation systems, which recognize the needs and constraints of pedestrians, and provide an optimized usefulness (usability and utility) to support pedestrian navigation. This article presents our recent efforts towards these concerns, mainly focusing on route planning and route communication.

\section{Introduction}

In daily life, people often encounter navigation problems when arriving at a new place, e.g. "what's the way from the train station to the city hall". Usually, people refer to maps for guidance or ask other people in the surrounding for advice.

In recent years, interest in using mobile phones to assist wayfinding is increasing. Mobile navigation systems are designed for this purpose. They often employ Global Positioning System (GPS) or other positioning methods for continuous tracking of mobile users, and thus provide users with real-time and in-situ route guidance (CoRONA \& W INTER 2001). Car navigation is the most successful application of mobile navigation systems. Recently, the increasing ubiquity of personal smart phones has triggered a move towards mobile pedestrian navigation systems.

Compared to car navigation systems, which are very mature and probably less complex, navigation systems for pedestrians are still in an early stage of development. Many of them adopt methods employed in car navigation systems. However, for pedestrians the degree of freedom in movement is larger than that for car drivers, and the typical resolution of space used for navigation is also larger (CORONA \& WINTER 2001). Therefore, information needs of pedestrians are quite different from those of 
car drivers. For example, in terms of route planning, car navigation systems often use shortest routes or fastest routes. However, according to Golledge (1995), pedestrians - especially when having enough time - might prefer different route qualities rather than shortness, e.g. simplicity, safety, attractiveness, and convenience. In terms of communicating route information (directions), car navigation systems usually provide metric route instructions together with simple maps. However, according to MichoN \& Denis (2001), when generating route descriptions, people often refer to landmarks located along the route. Therefore, for designing pedestrian navigation systems, approaches of car navigation systems are not suitable.

This article presents our recent efforts addressing key challenges of designing pedestrian navigation systems. We aim to develop pedestrian navigation systems that are human-centred and thereby effectively facilitate users' navigation tasks. In order to achieve the goal, several interdisciplinary projects are implemented. These projects integrate research methods of spatial cognition and human navigation as well as usability studies and mainly focus on two key elements of navigation systems, i.e. route planning and route communication. In this article, a selection of methods and findings of these projects is presented.

\section{Human navigation and mobile navigation systems}

\subsection{Human navigation}

Montello (2005, p. 257) defines navigation as "coordinated and goal-directed movement through the environment by organisms or intelligent machines". According to Downs \& STEA (1977), navigation includes four processes: orientation, planning the route, keeping on the right track, and discovering the destination. Similarly, MonTELLO (2005) conceptualizes navigation as consisting of wayfinding and locomotion. Wayfinding is the planning and decision-making part of navigation, and it requires that we know where to go and how to get there. Wayfinding needs a destination, a place we want to reach. This destination is often located outside of our immediate surroundings. Therefore, in order to make route choices, internal memories, or external artefacts such as maps, are often employed during wayfinding. In contrast, locomotion requires that we can move along a planned route efficiently, as well as coordinating body movements in respect to surroundings. During route following (locomotion), people constantly monitor their local surroundings. Objects of the real world (local surroundings) are compared with memories stored internally or external artefacts such as maps for route confirmation ("keeping the right track") (GARTNER \& HiLler 2009). This process continues until the destination is reached.

In summary, successful navigation requires knowing where to go and how to get there (the wayfinding part of navigation); it also requires moving along an intended route in an intended direction without having accidents or getting unnecessarily delayed (the locomotion part of navigation) (MONTELLO \& SAS 2006). 


\subsection{Basic elements and research questions in mobile pedestrian navigation systems}

Mobile navigation systems are designed to facilitate users' navigation tasks in unfamiliar environments. Navigation systems usually consist of three modules: positioning, route planning and route communication (HuANG \& GARTNER 2009).

The positioning module determines the current location of the user. For outdoor navigation, GPS is often used. Map matching algorithms are often employed to improve poor GPS accuracy in urban environments. For indoor navigation, other technologies are used, such as WiFi, Bluetooth, and radio-frequency identification (RFID). The positioning module helps to solve problems in wayfinding and locomotion: "Where am I?"

The route-planning module focuses on computing the most appropriate route from an origin to a destination, and thereby assists users in solving the main problem in wayfinding: "How can I get to the destination?".

When a route is chosen, users should move along the route to reach the destination. The route communication module aims at exploring technologies to convey route information (directions) effectively. Often, based on the real-time location information provided by the positioning module, in-situ route information can be provided during users' locomotion.

This article focuses on route planning and route communication.

\subsubsection{Route planning}

Route planning aims to compute the "best" route from an origin to a destination. Car navigation usually employed fastest routes and shortest routes. However, some other route-choice criteria are often used for pedestrian navigation, such as attractiveness, simplicity, and safety (GoLLEDGE 1995). Some previous field experiments carried out in our group confirmed this argument and suggest offering different (but not too many) routes with different characteristics, so that a user can choose the "best" route by herself/himself (GARTNER \& RADOCZKY 2006).

In order to provide routes with different characteristics, navigation systems need measures to characterize routes concerning these aspects. Current road network datasets, however, often lack these kinds of measures and thus make it hard to calculate other kinds of routes except the shortest and the fastest one. There is some research focusing on calculating different "best" routes based on existing street datasets, such as: routes with a minimal number of turns and routes with minimal angle (WINTER 2002), routes with least instruction complexity (DuCKHAM \& KULIK 2003), and reliable routes minimizing the number of complex intersections with turn ambiguities (HAQUE et al.2007). However, route qualities such as simplicity, safety, attractiveness, or convenience are difficult to measure and cannot be accurately reflected by geometrical characteristics of the road network (e.g. the number of turns, and the degree of angle). 


\subsubsection{Route communication}

When a suitable route between an origin and a destination is chosen, it needs to effectively guide users to move along the route. Car navigation systems often provide drivers with metric route instructions, such as: "Drive straight for 1.2 kilometres". However, this kind of instructions is not appropriate for pedestrians since pedestrians mainly use environmental information (such as landmarks) for navigation and do not rely much on metric information (Michon \& DeNis 2001, Rehrl et al. 2009). Semantic route instructions enriched with landmarks should be provided.

In addition, as pedestrians are free from driving tasks, more detailed information can be provided. Also besides verbal instructions, some other interface technologies, such as mobile maps, images and $3 \mathrm{D}$, may also play important roles in conveying route information for pedestrians. Recently, mobile augmented reality (AR), which enhances a real world camera view with virtual information overlays, is another approach for conveying route information (REHRL et al. 2011). WALTHER-FRANKS (2007) shows that AR is very suitable for navigation, as it puts route instructions directly into the real visual context of a user.

In order to guide users from an origin to a destination effectively, these techniques need to be carefully designed and evaluated. However, common rules or standards for using these techniques for route communication are not defined yet (GARTNER 2004). Related findings of spatial cognition and human navigation together with cartographic communication and usability studies might help to address this issue.

Spatial knowledge acquisition, which is needed to build mental representations of space, is another important aspect of route communication. These mental representations are essential for wayfinding and other spatial tasks (GARTNER \& HilLER 2009). With sufficient spatial knowledge about an environment, people can still find their way when navigation systems fail (e.g. out of battery). Currently, more and more people are relying (or even over-relying) on navigation systems. Therefore, it is essential to understand how these systems affect the acquisition of spatial knowledge during navigation. It might be useful to explore novel interface techniques facilitating both navigation and spatial knowledge acquisition.

\section{Selected contributions to the concept of human-centred mobile pedestrian navigation systems}

In order to ensure the usefulness (utility and usability), navigation systems should be human-centred. Human-centred pedestrian navigation systems understand the needs, limitations and constraints of pedestrians and integrate this understanding into the design process (WIKIPEDIA 2011).

Some interdisciplinary projects have been implemented at the Vienna University of Technology to concept our ideas on human-centred pedestrian navigation systems. We study the needs and constraints of pedestrians, and incorporate the understanding into 
the design of pedestrian navigation systems. In this way, we aim to develop navigation systems that are adapted to pedestrians and have an optimized utility and usability. In the following, a selection of proposed methods and results from our recent projects is presented. Sections 3.1 and 3.2 focus on pedestrian route planning and provide models to enable pedestrians with adaptive route recommendations. Sections 3.3 and 3.4 focus on route communication and aim at exploring interface technologies to convey route information effectively. These findings and results can be integrated to design human-centred pedestrian navigation systems.

\subsection{Collective intelligence-based routing: Asking others for route advice}

\subsubsection{Motivation and methodology}

A collective intelligence-based routing model is motivated by the "social strategy" of navigation: In daily life, we often ask more experienced people in the surrounding for orientation and route advice. In recent years also a trend towards incorporating the "participation" notion of Web 2.0 into Location Based Services has become obvious. Users are encouraged to annotate their personal feelings (i.e. user-generated content, UGC) to physical places when using the systems. Research has shown that experiences of other users in similar contexts can help current users to get answers to their problems (WeXelblat 1999).

Based on the motivation mentioned, a model for collective intelligence-based routing is proposed. We call it this name because it provides routes by aggregating other people's UGCs reflecting their collective intelligence. This model was initially tested in a smart environment set up in the UCPNavi (Ubiquitous Cartography for Pedestrian Navigation) project. During navigation in the smart environment, users were encouraged to give ratings for every decision point (DP) to indicate their perceived complexity of making the correct decision (choosing the correct road/branch to follow) at this DP. In order to make use of these ratings, we represent these ratings in a restricted pseudo-dual graph (WINTER 2002) and revise DIJKSTRA's algorithm for route calculation (HUANG \& GARTNER 2011). In this way, two kinds of routes are provided: the least complex route and the length-complexity-optimized (LCO) route.

1) The least complex route is defined as the route with the least complexity rating between an origin and a destination. A route often consists of a sequence of DPs. The overall complexity rating of the entire route is simply calculated as the sum of the complexity ratings of each DP. Thus, the least complex route is the route with the minimal overall complexity rating. For every DP, complexity ratings by different users may be available. We average these ratings to represent the collective intelligence-based complexity rating for that DP. Therefore, the least complex route can be considered as "the route that most people thought to be the least complex".

2) The LCO route considers both complexity ratings of DPs and the Euclidean distance of route segments. It tries to find an optimal trade-off between the least complex route and the shortest route. 


\subsubsection{Evaluation and simulation}

Some simulations using the street network of the First District of Vienna [Innere Stadt, Wien] were designed to evaluate the collective intelligence-based routing model. We simulated the DP ratings by simply considering the number of available alternatives at DPs. Therefore, for each pair of origin and destination, the least complex route and the LCO route can be computed by the proposed model. The corresponding shortest route was also calculated as a benchmark. Figure 1 shows a typical example of different routes between an origin and a destination, comparing the least complex route, the $\mathrm{LCO}$ route, and the shortest route. The LCO route achieves an optimal trade-off between complexity rating and length.

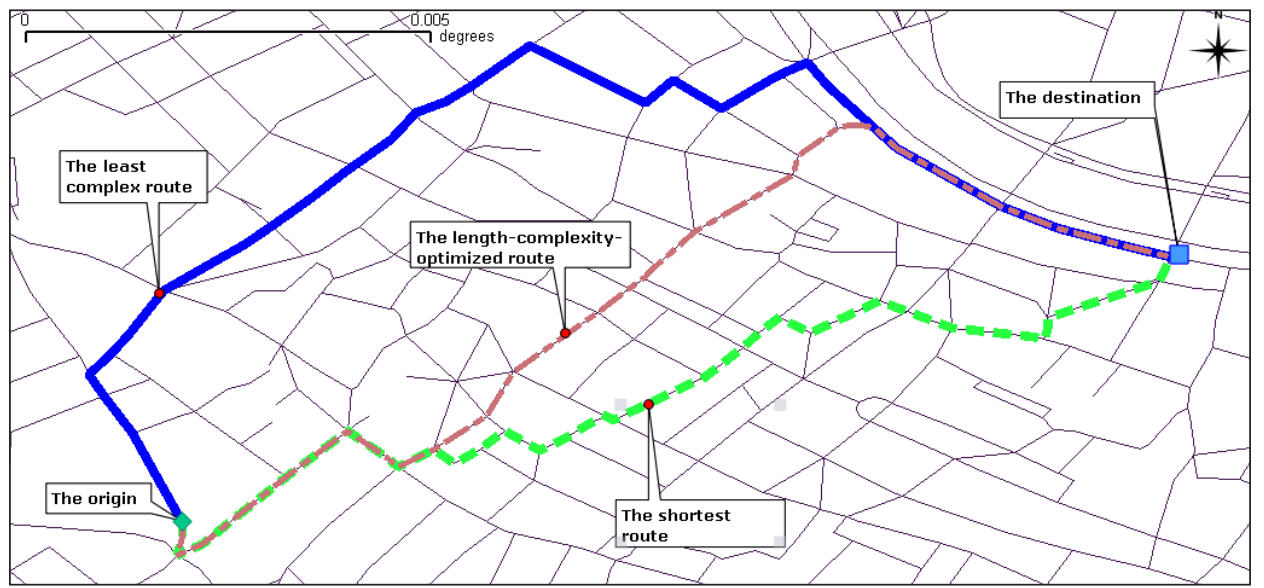

Fig. 1: Comparison of the least complex route, the length-complexity-optimized (LCO) route, and the shortest route between an origin and a destination, complexity rating (38 vs. 49 vs. 58), and length (1,603 metres vs. 1,334 metres vs. 1,266 metres). The least complex route has a lower complexity rating, but it is also longer. The LCO route is an optimal trade-off between the least complex route and the shortest route.

In order to study how these routes lead to different navigation performances, simulated navigators (agents) imitating some simple behaviour of human navigators were implemented to traverse these routes. Figures 2 and 3 depict the comparison of navigation performances when using these different routes. Two main metrics were used to measure the performances: percentage of failed travels,$^{2}$ and the actual distance travelled when successfully arriving at the destination (including distance travelled due to errors).

\footnotetext{
2) If the agent has made ten errors during following the route (e.g. choosing a wrong outgoing branch at an intersection), its travel is considered as a failed travel.
} 


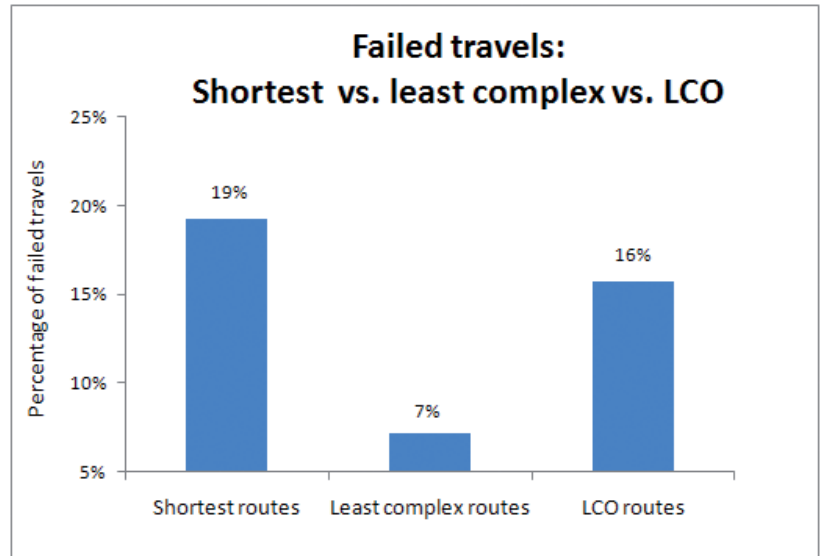

Fig. 2: Comparison of failed travels when using different routes

Figure 2 shows that taking least complex routes leads to the best performance in terms of the percentage of failed travels, followed by using LCO routes and using shortest routes $(7 \%$ vs. $16 \%$ vs. $19 \%)$. This means that using least complex routes raises the chance of reaching a destination (successful navigation).

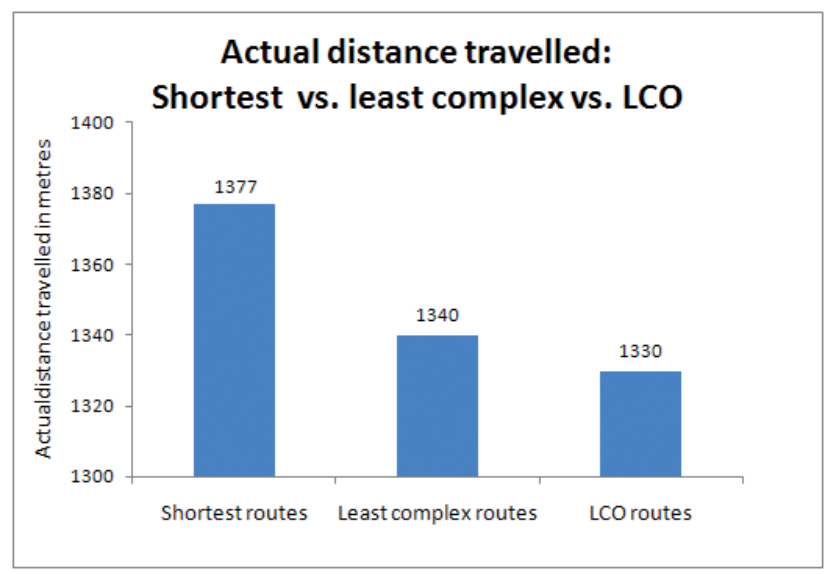

Fig. 3: Comparison of the actual distance travelled when using these different routes

In terms of the actual distance travelled (Fig. 3), using LCO routes leads to the best performance, followed by using least complex routes, and using shortest routes (1,330 metres vs. 1,340 metres vs. 1,377 metres). That means using LCO routes leads to shorter travel distance compared to using the other two kinds of routes.

Results considering other aspects of wayfinding performance can be found in HuANG \& GARTNER (2011). Compared to shortest routes, the collective intelligence-based routes (i.e. least complex routes, and LCO routes) show a significant improvement of route 
quality (i.e. with less complexity rating), thereby more effectively supporting users' navigation tasks (i.e. more chances of reaching destinations, fewer errors made, and shorter actual distance travelled).

\subsubsection{Discussion}

The collective intelligence-based routing model relies on other users' feedback reflecting their navigation experiences in the environment. If other users in similar context consider a specific route as attractive, probably this route can also be viewed as attractive for the current user in the current context. From this aspect, other users can help the current user to define/measure route qualities. The proposed model works with existing road datasets, and can be extended to offer routes with different characteristics.

It is also crucial to note that although the proposed approach is motivated by the "social strategy" of daily navigation, there are some differences. In daily life, people in the surrounding area may give biased route advice that only fits to themselves and may not be suitable to the current navigator. It is also unrealistic to ask many people for advices. The proposed approach can alleviate these problems above. It employs the methodology of Web 2.0, and aggregates/averages similar users' opinions to make appropriate route recommendations.

\subsection{Towards a typology of pedestrian behaviour}

Creating a typology of pedestrian behaviour is part of the research goals of the UCPNavi project. It aims to identify and describe typical classes of pedestrian spatial behaviour as well as route choice behaviour and to determine characteristic attributes for each class. It is expected that models of pedestrian mobility styles might offer a reliable basis for providing type-related route recommendations in pedestrian navigation systems (Millonig \& Gartner 2011).

In order to derive the pedestrian typology, a triangulation approach was employed (Millonig \& Gartner 2009). Firstly, unobtrusive observations and standardized interviews were used to identify basic types of pedestrian spatio-temporal behaviour. This initial typology was then verified by combining localization and detailed semistandardized interview techniques. Finally, results of both empirical phases were integrated to develop a typology of pedestrian walking styles.

This triangulation approach was used to derive pedestrian typology both outdoor and indoor: in a shopping street and a shopping mall, both in Vienna. In the following, we present the results of a data analysis based on more than 100 participants in a shopping street. Five types of motion behaviour were identified: convenient, hedonistic, discerning, swift and utilitarian. In addition, key determinants, characteristics and information needs of each type were identified.

The results can be used in mobile navigation systems to provide type-based route recommendations. For example, as swift shoppers do not want to spend more time than necessary in a shopping environment, navigation systems could provide shortest 
routes for allowing them to complete their tasks as quickly as possible. For convenient shoppers, navigation systems could suggest pleasant routes passing the most attractive shops and nice rest areas. See Millonig \& Gartner (2011) for a detailed discussion about the application of the results above.

With the models suggested in sections 3.1 and 3.2, adaptive route recommendations can be provided to pedestrians. The following two sections will discuss our findings on how to guide pedestrians to follow a chosen route.

\subsection{Semantic route instructions}

Different interface technologies can be used to communicate/convey route information (directions) to pedestrians, such as maps, textual or verbal information, images, videos, and 3D. In this section, we will focus on voice-based route guidance (verbal route instructions).

In order to support pedestrian navigation, verbal instructions need to be designed carefully. The SemWay project, implemented by Salzburg Research, Vienna University of Technology and industry partners, proposed a concept of semantic wayfinding (ReHrl et al. 2009). The aim was to study how people describe the world and routes. This understanding was then used to develop a formal model of navigation language.

The model was derived from empirical data, i.e. verbal descriptions of route choices collected in the context of two in-situ experiments in the cities of Salzburg and Vienna. In total, 7,151 propositions from 20 participants for 100 decision points were collected. They were processed and classified using Denis's classification (DenIs 1997). Three kinds of concepts forming route instructions were extracted and modelled (REHRL et al. 2009): motion concepts (a set of re-occurring verbs describing motion patterns, such as turn, walk, pass and cross), direction concepts (a set of re-occurring spatial relations that can be used to anchor motion with landmarks, such as along, in, out, direction of, to, through, and through between), and landmark concepts (reference entities along different route segments). Based on the model, semantic route instructions instead of metric instructions can be provided, for example, "walk straight, pass the theatre, and walk to the crossing" instead of "walk straight for 103 metres".

REHRL et al. (2010) designed a field experiment to compare metric instructions and semantic instructions in the context of mobile pedestrian navigation. The results show that both kinds of instructions led to similar navigation performance in terms of walking time and decision errors. However, participants had more confidence in their decisions at intersections when using semantic instructions, and clearly preferred this kind of instructions.

\subsection{Spatial knowledge acquisition with mobile maps, augmented reality (AR) and voice}

As mentioned above, route information can be communicated via maps, verbal instructions, 3D, AR, etc. When evaluating the suitability of different interface tech- 
nologies in conveying route information, two key questions need to be considered: (1) How do these interface technologies lead to different wayfinding performances (e.g. how many errors do users make during navigation, how long do users spend to navigate along a route)? (2) How do these interface technologies affect the acquisition of spatial knowledge? For some findings on the first question, refer to the report of the previous project NAVIO (RADOCZKY 2004).

This section presents our findings on the aspect of spatial knowledge acquisition with different interface technologies (mobile maps, AR, and voice). It is part of the ways2navigate project, implemented by Salzburg Research, Vienna University of Technology, FACTUM, TraffiCon and WalkSpace Mobilität, aiming to compare mobile maps, AR, and voice for route communication in the context of GPS-based pedestrian navigation.

Three navigation prototypes, implementing mobile map-based, AR-based and voicebased guidance, respectively, were carefully developed by integrating recent findings on conveying route information from literature (REHRL et al. 2011). Figure 4 shows a screenshot of the map-based prototype, including an egocentric view, distinction between the past and future path, automated adaptation to real-time location, highlighted landmarks, zooming and panning function, etc. Figure 4 also depicts a screenshot of the AR-based prototype, showing a real-world camera view, route overlay, street names and relevant landmarks. Based on the findings of the SemWay project (refer to section 3.3), semantic route instructions instead of metric route instructions were provided in the voice-based prototype.
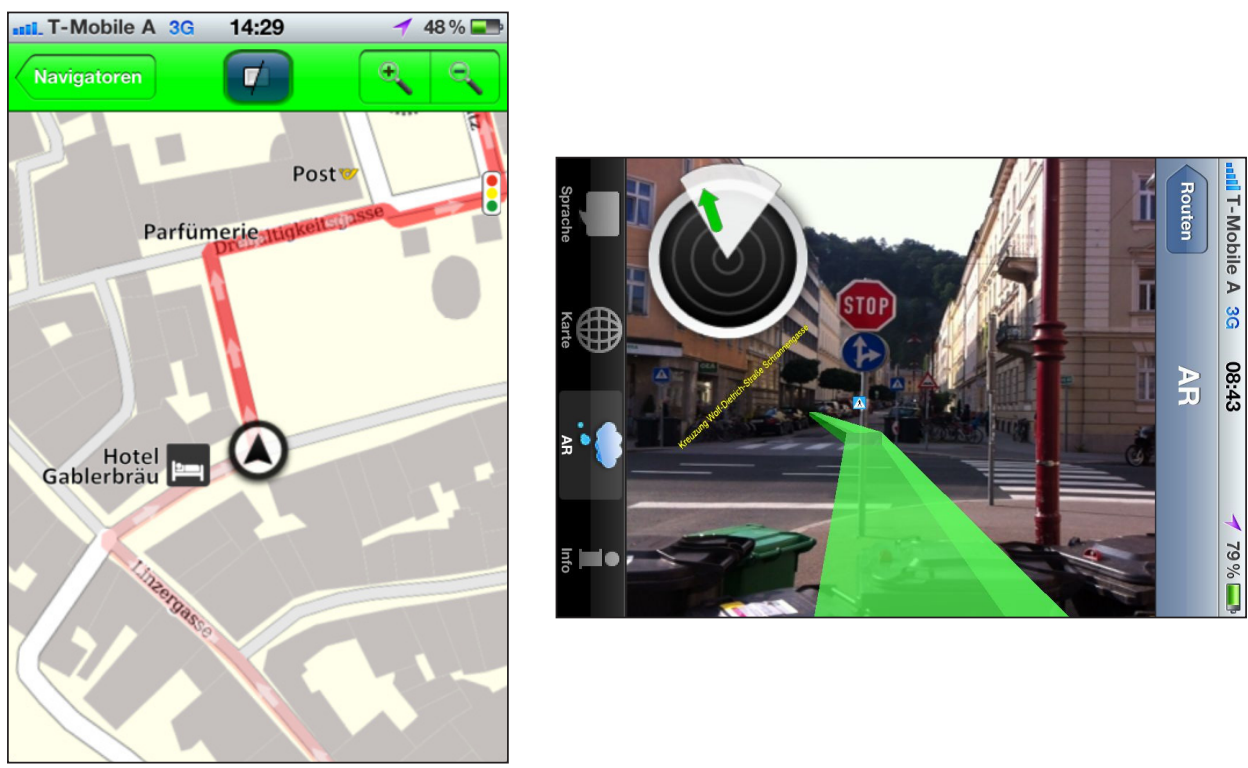

Fig. 4: Screenshots of the map-based navigation system (left), and the AR-based navigation system (right) 
A field test with 17 participants (nine female and eight male, with a mean age of 43 years, ranging from 21 to 73 years) was carried out in the city centre of Salzburg (Austria) to investigate how these three navigation prototypes affect the acquisition of spatial knowledge during navigation. Participants were randomly assigned to one of the three groups, mobile map $(n=6), A R(n=7)$, and voice $(n=4)$. The male/female ratios were similar in the three groups. Each participant was asked to navigate along the pre-defined route by using the corresponding navigation system. When they reached the end of the route, they were asked to recognize landmarks from a photo album (landmark recognition task), recall how they turned at each intersection (route direction task), and place the chosen photos on an A4 map of the area (landmark placement task).

Figure 5 shows the average error in the route direction task. AR users and map users performed slightly better in recalling turning directions at intersections (with $34 \%$ errors for AR users, and $37 \%$ errors for map users) compared to voice users with $40 \%$ errors. However, there was no significant difference among the three navigation kinds $[\mathrm{F}(2,14)=0.09, \mathrm{p}=0.91]$.

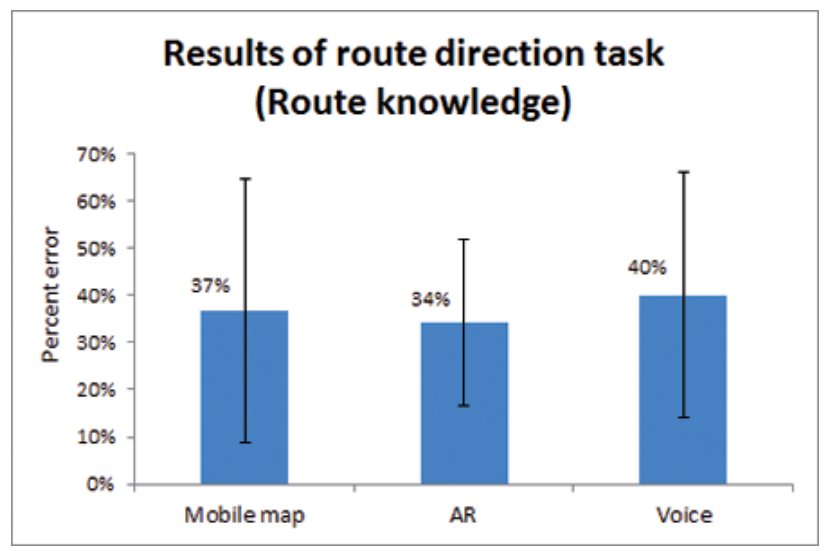

Fig. 5: Results of the route direction task (mean error); vertical bars denote $95 \%$ confidence intervals

Similar results can be found for the landmark recognition task and the landmark placement task (HuANG et al. 2011). In summary, the results show that for all three kinds of spatial knowledge acquisition, navigation by AR had a better performance than navigation by map and navigation by voice. However, the differences among the three navigation kinds were not significant at the $5 \%$ level. It is also worth noting that in all three navigation kinds, participants perform quite poor in all of the three tasks, not significantly different from chance.

We interpreted and analysed the results by using the "active encoding principle" proposed by MüNZER et al. (2006): Only information that is "actively" processed during the primary navigation activity is learned and remembered. Based on the interpretation, we conclude that since mental efforts involved in GPS-based pedestrian navigation are all reduced to a minimum, these three interface technologies lead to comparably poor performances in spatial learning during navigation (HuANG et al. 2011). 
Results of the field test lead to several research questions: Is it important/useful to have a navigation system that not only effectively guides users from A to B, but also enables them to acquire spatial knowledge during navigation? If yes, how can we design this kind of systems? Related findings of spatial cognition and human navigation together with cartographic communication and usability studies will be integrated to address these questions.

\section{Conclusions and outlook}

Recent years have seen an increasing interest in mobile pedestrian navigation systems. However, these systems are still in early stages of development. In this article, basic elements and research questions for mobile pedestrian navigation systems were discussed and compared with car navigation systems. We proposed that in order to ensure their utility and usability, pedestrian navigation systems should be humancentred and aware of the needs, limitations and constraints of pedestrians. Findings and results from our recent research projects addressing these concerns were presented. It is expected that these results will help to develop human-centred pedestrian navigation systems to effectively support pedestrians' navigation tasks.

Context-awareness plays a key role in Location Based Services. Our next step is to explore context-aware adaptation for pedestrian navigation including context and user modelling, adaptive route planning, as well as adaptive route communication.

\section{References}

Corona B., Winter S. (2001), Guidance of car drivers and pedestrians. Technical report, Inst. for Geoinformation and Cartogr., Vienna Univ. of Technology.

DenIs M. (1997), The description of routes: A cognitive approach to the production of spatial discourse. In: Cahiers de Psychologie Cognitive, 16, pp. 409-458.

Downs R.M., Stea D. (1977), Maps in minds: Reflections on cognitive mapping. New York, Harper \& Row.

Ducкham M., Kulik L. (2003), "Simplest" paths: Automated route selection for navigation. In: Kunn W., Worboys M., TimpF S. (eds.), COSIT 2003, pp. 169-185. Berlin - Heidelberg, Springer.

Gartner G. (2004), Location-based mobile pedestrian navigation services - the role of multimedia cartography. In: Proc. of $1^{\text {st }}$ ICA UPIMap2004, Tokyo.

GarTner G., Hiller W. (2009), Impact of restricted display size on spatial knowledge acquisition in the context of pedestrian navigation. In: Gartner G., Rehrl K. (eds.), Location Based Services and TeleCartography II, pp. 155-166. Berlin - Heidelberg, Springer.

Gartner G., Radoczky V. (2006), About the Role of Cartographic Presentation for Wayfinding. In: Stefanakis E. et al. (eds.), Geographic Hypermedia Concepts and Systems (= Lecture Notes in Geoinformation and Cartography), pp. 381-398. Berlin - Heidelberg, Springer. 
Golledge R.G. (1995), Path Selection and Route Preference in Human Navigation: A Progress Report. In: Frank A.U., KuHn W. (eds.), Spatial Information Theory - A Theoretical Basis for GIS, pp. 207-222. Berlin - Heidelberg, Springer.

Haque S., Kulik L., Klippel A. (2007), Algorithms for reliable navigation and wayfinding. In: BARKowsky T. et al. (eds.), Spatial Cognition V, pp. 308-326. Berlin - Heidelberg, Springer.

Huang H., Gartner G. (2009), Using Activity Theory to identify relevant context parameters. In: Gartner G., Rehrl K. (eds.), Location Based Services and TeleCartography II - from Sensor Fusion to Context Models (= LNG\&C), pp. 35-45. Berlin - Heidelberg, Springer.

Huang H., Gartner G. (2011), Collective Intelligence Based Route Recommendation for Assisting Pedestrian Wayfinding in the Era of Web 2.0. Accepted for Journal of Location Based Services.

Huang H., Schmidt M., Gartner G. (2011), Spatial knowledge acquisition with mobile map, augmented reality, and voice. Internal report, Vienna Univ. of Technology.

Michon P.E., Denis M. (2001), When and why are visual landmarks used in giving directions? In: Montello D.R. (ed.), Spatial Information Theory, pp. 243-259. Berlin, Springer.

Millonig A., Gartner G. (2009), Ways of Walking - Developing a Pedestrian Typology for Personalised Mobile Information Systems. In: Gartner G., Rehrl K. (eds.), Location Based Services and TeleCartography II - from Sensor Fusion to Context Models (= LNG\&C), pp. 79-94. Berlin - Heidelberg, Springer.

Millonig A., Gartner G. (2011), Identifying motion and interest patterns of shoppers for developing personalised wayfinding tools. In: Journal of Location Based Services, 5, 1, pp. 3-21.

Montello D.R. (2005), Navigation. In: Shah P., Miyake A. (eds.), The Cambridge handbook of visuospatial thinking, pp. 257-294. Cambridge, Cambridge Univ. Press.

Montello D.R., SAS C. (2006), Human factors of wayfinding in navigation. In: KARwowsKi W. (ed.), International encyclopedia of ergonomics and human factors, $2^{\text {nd }}$ ed., pp. 2003-2008. London, CRC Press/Taylor \& Francis, Ltd.

MünZer S. et al. (2006), Computer Assisted Navigation and the Acquisition of Route and Survey Knowledge. In: Journal of Environmental Psychology, 26, pp. 300-308.

Radoczky V. (2004), Literature Review and Analysis about Various Multimedia Presentation Forms. Internal report, Vienna Univ. of Technology.

RehrL K. et al. (2009), An analysis of direction and motion concepts in verbal descriptions of route choices. In: HornsBy K.S. et al. (eds.), COSIT 2009, pp. 471-488. Berlin - Heidelberg, Springer.

Rehrl K., Häusler E., Leitinger S. (2010), Comparing the effectiveness of GPS-enhanced voice guidance for pedestrians with metric- and landmark-based instruction sets. In: FABriKant S.I. et al. (eds.), GIScience 2010, pp. 189-203. Berlin - Heidelberg, Springer.

ReHrL K. et al. (2011), Pedestrian navigation with augmented reality, voice and digital map: results from a field study assessing performance and user experience. Accepted for Proc. of Location Based Services 2011, Vienna, Austria.

W ALther-Franks B. (2007), Augmented reality on handhelds for pedestrian navigation. Master thesis, Univ. of Bremen.

Wexelblat A. (1999), Footprints: Interaction history for digital objects. PhD Thesis, Massachusetts Inst. of Technology.

WIKIPEDIA (2011), User-centered design. - http://en.wikipedia.org/wiki/User-centered_design (accessed on 08.2011)

WINTER S. (2002), Modelling costs of turns in route planning. In: GeoInformatica, 6, 4, pp. 345-361. 